\title{
Entoto Twining Telescopes: first facilities in East Africa for astronomy research
}

\author{
Solomon Belay Tessema ${ }^{1}$ and Leonid Berdnikov ${ }^{1,2}$ \\ ${ }^{1}$ Astronomy and Astrophysics Research Division, Entoto Observatory and Research Center, \\ P.O. Box 33679 Addis Ababa, Ethiopia \\ email: tessemabelay@gmail.com \\ ${ }^{2}$ Sternberg Astronomical Institute, Lomonosov Moscow State University, \\ Universitetskii pr. 13, Moscow, 119992 Russia \\ email: leonid.berdnikov@gmail.com
}

\begin{abstract}
Recently Entoto observatory and research Center launched two 1-m twining telescopes, located on the Entoto mountain in the suburb of Addis Ababa. These telescopes, equipped with four CCDs and echelle spectrograph, will be used for observations of the different types of variable stars, X-ray binaries, double stars, star clusters, exo-planets etc.
\end{abstract}

Keywords. Entoto observatory, optical telescopes

\section{Introduction}

The Entoto Observatory (EO) has two 1 meter aperture alt-az telescopes with each having a primary mirror supported by Active-Optics actuators and two Nasmyth foci. The 1-m telescopes, equipped with four CCDs: SBIG STL $11000 M(4000 \times 2672,9 \mu m)$, SBIG STL $1001 E(1024 \times 1024,24 \mu m)$, ANDOR iXon3-888 $(1024 \times 1024,13 \mu m)$ and FLI PL $4301 \mathrm{E}-1(2048 \times 2048,24 \mu \mathrm{m})$ as well as with Fibre Linked Echelle Astronomical Spectrograph (FLECHAS) with spectral range from 389 to $762 \mathrm{~nm}$ (on CCD detectors of $2048 \times 2048$ pixels, $15 \mu \mathrm{m}$ pixel size) and Spectral peak resolution $R\left(\frac{\lambda}{\Delta \lambda}\right) \sim 37000$, are ideal tools for studying variable stars, binary stars, X-ray binaries, star clusters, exoplanets, structure and kinematics of the disk and halo of our Galaxy etc. We present here the very first results of our observations - light curves of V504 Sgr which is a RR Lyrae type star with a period 0.4 days. During two successive nights in May 2015 we conducted VRI differential observations of V504 Sgr with the CCD "Andor iXon3-888". Seeing was changing from 1.6 to 2.3 arcsec. The light and color curves for V504 Sgr are presented in Fig. 1

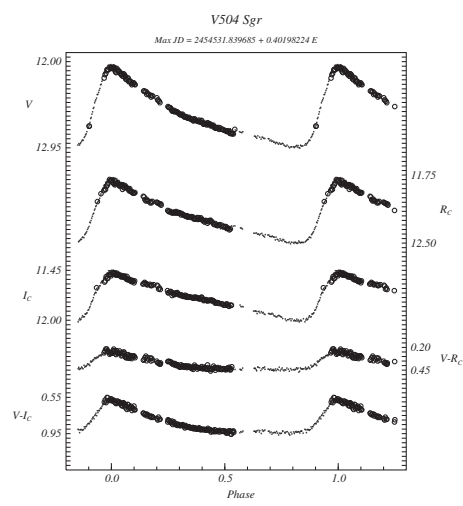

Figure 1. Light and color curves for V504 Sgr. 